\title{
Effective Potential Improvements, $\epsilon$-expansions, and the Electroweak Phase Transition
}

\author{
G. Amelino-Camelia ${ }^{a}$ \\ ${ }^{a}$ Center for Theoretical Physics, Laboratory of Nuclear Science and Department of Physics, \\ Massachusetts Institute of Technology, Cambridge, Massachusetts 02139, USA.
}

Two recently proposed approaches to the study of the electroweak phase transition are discussed.

\section{INTRODUCTION}

Recently, there has been considerable interest in the "electroweak phase transition" (the temperature induced symmetry-changing phase transition[1] of the standard electroweak model), especially in connection with the possibility of dynamical generation of the baryon asymmetry. An appropriate tool for the investigation of this type of phenomena is the finite temperature effective action [2] $\Gamma_{T}(\phi(x))$, where $\phi(x)$ is a trial vacuum expectation value of a scalarm quantum field of the theory. $\Gamma_{T}(\phi(x))$ is the generating functional of the one-particle irreducible Green's functions, and therefore encodes all physical information about the theory, but unfortunately, its evaluation is extremely difficult.

An important simplification can be achieved by considering position independent trial vacuum expectation values, $\phi(x)=$ constant $=\phi$. This leads to the introduction of the finite temperature effective potential $V_{T}(\phi)$, which is related to $\Gamma_{T}(\phi(x))$ by

$V_{T}(\phi) \equiv-\frac{\left[\Gamma_{T}(\phi(x))\right]_{\phi=c o n s t a n t}}{\int d x}$.

$V_{T}(\phi)$ only encodes static|2 information about the theory (it is the generating functional of oneparticle irreducible Green's functions at zero external momentum), but still it can be valuable in (at least preliminary) investigations of temperature induced phase transitions.

In spite of the simplification that follows from

\footnotetext{
${ }^{1} \mathrm{~A}$ more general definition of the effective action can be given 2, but for the purpose of this talk it is sufficient to consider this $\Gamma_{T}(\phi(x))$.
}

considering position independent trial vacuum expectation values, the evaluation of the effective potential $V_{T}(\phi)$ is still rather difficult. It is not hard (in the imaginary time formalism of finite temperature field theory) to set up a loop expansion[3] of $V_{T}(\phi)$ in terms of vacuum-to-vacuum one-particle irreducible Feynman diagrams whose lines represent the tree-level propagator, and to evaluate (in some cases even analytically) the first diagrams of this expansion, but near the critical temperature the expansion is affected by infrared problems which render non-negligible the contributions of some classes of multi-loop diagrams. Therefore, studies of temperature induced phase transitions cannot rely on approximations of $V_{T}(\phi)$ which are obtained by truncating this "ordinary loop expansion" (i.e. the loop expansion discussed in Ref.(3]).

Several alternative techniques have been proposed for a more reliable analysis of temperature induced symmetry-changing phase transitions. In the following, I discuss some aspects of two of these techniques. First, I consider improvements in the approximation scheme for the effective potential based on selective summations of multiloop diagrams, and, then, I discuss a technique for the study of temperature induced symmetrychanging phase transitions that uses renormalization group and $\epsilon$-expansion.

\section{SELECTIVE SUMMATION OF MULTI-LOOP DIAGRAMS}

Several improved approximations of the effective potential based on selective summations of 
multi-loop diagrams have been discussed in the literature (see, for example, Refs.[4-13]); in particular, a way to perform systematically such summations [8-13] can be found within the formalism of the effective potential for composite operators 2,14 $V_{T}(\phi, G)$.

$V_{T}(\phi, G)$ is a generalization of $V_{T}(\phi)$ which, besides depending on a trial vacuum expectation value of the one-point-function $(\phi)$, also depends on a trial vacuum expectation value of the twopoint-function $(G)$, and is related to $V_{T}(\phi)$ by

$V_{T}(\phi) \equiv V_{T}\left(\phi, G_{0}\right)$,

where $G_{0}$, which can be shown to be the full propagator of the theory, is the solution of the stationary requirement

$\left[\frac{\delta V_{T}(\phi, G)}{\delta G}\right]_{G=G_{0}}=0$.

Eq.(2) implies that, by fixing $G=G_{0}$ in the loop expansion of $V_{T}(\phi, G)$ discussed in Ref. 14], one can obtain an "improved loop expansion" of $V_{T}(\phi)$, in terms of vacuum-to-vacuum twoparticle irreducible Feynman diagrams whose lines represent the full propagator $G_{0}$. It can be shown 8, 10, 12, 14 that approximations of $V_{T}(\phi)$ which are obtained by truncating this improved loop expansion correspond to the resummation of the classes of multi-loop diagrams of the ordinary loop expansion which give the most important contributions near the critical temperature. This gives [10] an important tool for the study of "strongly first order" phase transitions?", and therefore renders possible the test of the most studied scenario 15] for "electroweak baryogenesis" (dynamical generation of the baryon asymmetry at the electroweak phase transition), which requires the electroweak phase transition to be strongly first order.

Preliminary investigations 16 of the electroweak phase transition using this method appear to lead to interesting results, most notably to the indication that (in agreement with recent numerical analyses[17-19]) the possibility of

\footnotetext{
${ }^{2}$ Here a phase transition is defined to be strongly first order if, at the critical temperature, the value of $\phi$ at which the symmetry-breaking minimum occurs is much greater than the product of the largest coupling of the theory and the temperature.
}

baryogenesis at the one Higgs doublet electroweak phase transition might be consistent with the present experimental lower limit on the Higgs mass.

Concerning the issue of reliability, it is important to realize that this and the other approaches based on selective summations of multi-loop diagrams are not useful for the study of second order or weakly first order phase transitions [10]. It should also be noted that there is no rigorous argument to indicate that the critical temperature (which is obviously also important in determining the value of the symmetry-breaking minimum at the critical temperature) can be reliably estimated within these approaches s]; however, the stability[8-11] of such estimates with respect to higher order corrections might be an indirect indication of their validity.

\section{3. $\epsilon$-EXPANSION}

Another technique that can be useful in the study of temperature induced symmetrychanging phase-transitions uses renormalization group and $\epsilon$-expansion 20,21]. This technique exploits the fact that at high temperatures the imaginary time formulation of finite temperature field theory can be written as an effective 3-dimensional field theory[2], with leading dependence on the temperature introduced by the renormalization group relations between the parameters of this 3-dimensional theory and their 4-dimensional counterparts. It is actually convenient 21] to consider such an effective theory in 4- $\epsilon$ dimensions, obtain results as an expansion in powers of $\epsilon$, and continue back to the 3dimensional theory only at the end, by taking the $\epsilon \rightarrow 1$ limit.

Several observables relevant for the understanding of the electroweak phase transition, and especially of electroweak baryogenesis, have been computed 21] using this " $\epsilon$-expansion method",

\footnotetext{
${ }^{3} \mathrm{As}$ discussed in Refs. 9. 10], selective summations of multi-loop diagrams should lead to reliable description of the effective potential for all values of $\phi$ greater than a certain $\tilde{\phi}$, but clearly, in determining the temperature at which the symmetry breaking minimum is degenerate with the symmetric $(\phi=0)$ one, it is necessary to accurately describe the effective potential also for $\phi \sim 0$.
} 
and it is believed that these results be reliable if the electroweak phase transition is weakly first order. In fact, the " $\epsilon$-expansion method" has been very successful in the study of the second order phase transition of the "scalar $\lambda \Phi^{4}$ theory", and it can therefore be reasonable to expect that it gives an accurate description of second order and weakly first order phase transitions. However, the $\epsilon$-expansion is actually asymptotic 21] (the terms of the expansion start growing in magnitude at orders $n \sim 1 / \epsilon$ ), and this renders somewhat doubtful the meaning of the results finally obtained taking the $\epsilon \rightarrow 1$ limit. In some cases the $\epsilon$-expansion is useless, because already the first terms of the expansion grow in magnitude. Even if one finds that, in a specific calculation, the first few terms of the expansion do behave perturbatively, this would still not prove (although it would be consistent with the fact) that the results of the " $\epsilon$ expansion method" for that calculation are reliable. This might be reason of concern especially for the case of first order phase transitions, for which (unlike the second order phase transition case) the accuracy of the " $\epsilon$-expansion method" has not been satisfactorily tested.

\section{CONCLUSIONS}

A lot remains to be understood about the electroweak phase transition. In this talk, I discussed some features and the expected (expectations which, however, rely on arguments that still need further investigation) reliability of two techniques which could be useful in the study of this phase transition. The most important observation is that it appears that these two techniques are somewhat complementary, one expected to be useful in the study of strongly first order phase transitions, and the other expected to be useful in the study of second order and weakly first order phase transitions. However, the results of some preliminary investigations $[4,16-19,21]$ can be interpreted as indicating that the (first order) electroweak phase transition might be neither strong enough to use confidently the method of selective summations of multi-loop diagrams contributing to the effective potential nor weak enough to use confidently the " $\epsilon$-expansion method". This fact should motivate additional and more rigorous analysis of the limits of validity of these techniques.

\section{ACKNOWLEDGEMENTS}

This work is supported in part by funds provided by the U.S. Department of Energy (D.O.E.) under cooperative agreement \#DEFC02-94ER40818, and by Istituto Nazionale di Fisica Nucleare (INFN, Frascati, Italy). I would like to thank J. March-Russell for a very stimulating conversation on the " $\epsilon$-expansion method", and G.R. Farrar for an informative conversation on the numerical results presented in Refs. 18, 19.

\section{REFERENCES}

1. D. Kirzhnits, JETP Lett. 15, 529 (1972); D. Kirzhnits and A. Linde, Phys. Lett. B 42, 471 (1972); D. Kirzhnits and A. Linde, JETP 40, 628 (1974).

2. R. Jackiw and G. Amelino-Camelia, in Proceedings of the Third Workshop on Thermal Field Theories and Their Applications, Banff, Canada, August 15-27, 1993, edited by F.C. Khanna, R. Kobes, G. Kunstatter, and H. Umezawa (World Scientific, 1994).

3. L. Dolan and R. Jackiw, Phys. Rev. D 9 (1974) 3320.

4. M. Dine, R.G. Leigh, P. Huet, A. Linde, and D. Linde, Phys. Rev. D 46, 550 (1992); Phys. Lett. B 283 (1992) 319.

5. M.E. Carrington, Phys. Rev. D 45 (1992) 2933.

6. P. Arnold and O. Espinosa, Phys. Rev. D 47 (1993) 3546.

7. C.G. Boyd, D.E. Brahm and S.D.H. Hsu, Phys. Rev. D 48 (1993) 4963.

8. G. Amelino-Camelia and S.-Y. Pi, Phys. Rev. D 47 (1993) 2356.

9. G. Amelino-Camelia and S.-Y. Pi, in Proceedings of the Third Workshop on Thermal Field Theories and Their Applications, Banff, Canada, August 15-27, 1993, edited by F.C. Khanna, R. Kobes, G. Kunstatter, and H. Umezawa (World Scientific, 1994). 
10. G. Amelino-Camelia, Phys. Rev. D 49 (1994) 2740.

11. G. Amelino-Camelia, in Proceedings of the Third Workshop on Thermal Field Theories and Their Applications, Banff, Canada, August 15-27, 1993, edited by F.C. Khanna, R. Kobes, G. Kunstatter, and H. Umezawa (World Scientific, 1994).

12. M. Quiros, hep-ph-9304284, based on talk given at IV Hellenic School on Elementary Particle Physics, Corfu, Greece, 2-20 Sep 1992.

13. J. Balakrishnan and I. G. Moss, Phys. Rev. D 49 (1994) 4113.

14. J.M. Cornwall, R. Jackiw, and E. Tomboulis, Phys. Rev. D 10, 2428 (1974).

15. For recent reviews, see: M. Dine, in proceedings of the Texas Symposium on Electroweak Baryon Number Violation, New Haven, USA, 1992; A.G. Cohen, D.B. Kaplan, and A.E. Nelson, Ann. Rev. Nucl. Part. Phys. 43 (1993) 27.

16. G. Amelino-Camelia, work in progress.

17. Contribution of G.R. Farrar to these proceedings.

18. K. Kajantie, K. Rummukainen, and M. Shaposhnikov, Nucl. Phys. B 407 (1993) 356.

19. K. Farakos, K. Kajantie, K. Rummukainen, and M. Shaposhnikov, Phys. Lett. B 336 (1994) 494.

20. J. March-Russell, Phys. Lett. B 296 (1992) 364.

21. P. Arnold and L.G. Yaffe, Phys. Rev. D 49 (1994) 3003. 